\title{
Nanoparticles and air pollutants as potential stimulators of asthmatic reaction
}

\author{
Shaza Abdulnasser Harfoush ${ }^{1}$, Juliane Nguyen ${ }^{2}$, Sebastian Heck ${ }^{1}$, Ahmed Mahdy ${ }^{3}$, Robert Bals ${ }^{3}$ and Quoc Thai Dinh ${ }^{1,3}$ \\ ${ }^{1}$ Department of Experimental Pneumology and Allergology, Saarland University Faculty of Medicine, Kirrberger Str.100, D-66421Homburg/Saar, Germany \\ ${ }^{2}$ Department of Pharmaceutical Sciences, School of Pharmacy and Pharmaceutical Sciences, 303 Kapoor Hall, SUNY Buffalo, 14214, New York, USA \\ ${ }^{3}$ Department of Internal Medicine V, Pneumology, Allergology and Respiratory Critical Care Medicine, Saarland University Faculty of Medicine, Kirrberger Str. \\ 100, D-66421 Homburg/Saar, Germany
}

\begin{abstract}
Nanotechnology offers great potential as an advanced therapeutic strategy for drug delivery and other biomedical applications. Nanoparticles (NPs) have individual characteristics that can be exploited for diagnostic or therapeutic purposes. They can access cells and interact with different cellular components including the nucleus. However, they can act as foreign materials that might aggravate different pathologies like chronic obstructive pulmonary disease (COPD) and asthma. Asthma is a chronic inflammatory obstructive lung disease characterized by hyperreactivity of the airways caused by diverse stimuli. Particulate matter (PM) and diesel exhaust nanoparticles $(\mathrm{DE})$ are nanoparticles that appear to have a number of serious side effects like respiratory tract irritation and carcinogenicity. They can also exacerbate allergic inflammation and asthma. This review summarizes the impact of air pollution particulates and provides an overview of different nanoparticles that affect our respiratory health and trigger airway inflammation.
\end{abstract}

\begin{abstract}
Abbreviations: NPs: Nanoparticles; COPD: Chronic obstructive pulmonary disease; PM: Particulate matter; DE: Exhaust nanoparticles; DEP: Diesel exhaust engines; AgNPs: Silver nanoparticles; $\mathrm{TiO}_{2} \mathbf{N P s}$ : Titanium dioxide nanoparticles; AHR: Airway hyperresponsiveness; ZnO NPs: Zinc oxide nanoparticles; BALF: Bronchoalveolar lavage fluid; CuO NPs: Copper oxide nanoparticles; SNPs: Silica nanoparticles; GONPs: Graphene oxide nanoparticles; $\mathrm{Nano}_{\mathbf{S i O}}$ : $\mathrm{Nano}$-silicon dioxide; CNTs: Carbon nanotubes; SWCNTs: Single-walled carbon nanotubes; DWCNTs: Double-walled carbon nanotubes; MWCNTs: Multi-walled carbon nanotubes
\end{abstract}

\section{Introduction}

Nanoparticles (NPs) have become a promising biomedical tool due to their physiochemical features such as biodegradability, biocompatibility, presumed minimal toxicity, and ease of modification [1]. NPs are defined as solid particulates or particle dispersions with sizes ranging from 1 to $100 \mathrm{~nm}$. They can permeate and remain active in different living tissues, cells, and the bloodstream, where they exert their effects [2]. Definition of the harmful effects of NPs is also important since they might induce tissue damage, inflammation, undesirable signal transduction, toxicological stress, and even creat genotoxicity [3,4]. Additionally, NPs can act as foreign materials that might aggravate different pathologies like chronic obstructive pulmonary disease (COPD) and asthma.

Asthma is a chronic inflammatory respiratory disease characterized by episodes of airway obstruction caused by a narrowing of the small airways in response to asthmatic "triggers" [5]. According to the World Health Organization (WHO), hundreds of millions of people suffer from asthma globally, and this number is rising; deaths from asthma have reached over 180,000 annually. Asthma attacks can vary from mild to life-threatening and mostly consist of a combination of symptoms including shortness of breath, cough, wheeze, chest pain, and chest tightness $[6,7]$. Many factors can trigger an asthma attack including allergens, infections, exercise, abrupt changes in the weather, or exposure to airway irritants such as tobacco smoke, engineered nanomaterials, and air pollutants.

Worldwide, air pollution and its particulate matter (PM) have received great attention due to their harmful effects on public health. Moreover, pollution in big cities is recognized as a major cause for asthma morbidity [7]. In our review, the toxicity of air pollutants including PMs and diesel exhaust (DE) nanoparticles will be redefined in order to quantify human exposure and improve population health.

\section{Nanoparticles trigger airway inflammation and exacer-} bate asthma

\section{Silver nanoparticles (AgNPs)}

Silver nanoparticles (AgNPs) have been widely exploited in biomedicine due to their excellent physicochemical properties $[8,9]$. They are the most commercialized nanomaterial, and they are used in a wide variety of products ranging from medical products to anti-odor textiles and paints.

${ }^{*}$ Correspondence to: Dinh QT, MD, Professor of Medicine, Department of Experimental Pneumology and Allergology, Saarland University Hospital and Saarland University Faculty of Medicine, Kirrberger Strasse, Geb. 61.4, 66421 Homburg/Saar, Germany, Tel: +496841 16 15561; Fax: +49 684116 47952; E-mail: thai.dinh@uks.eu

Key words: asthma, nanoparticles, airway inflammation, diesel exhaust nanoparticles, particulate matter, carbon nanotubes

Received: September 16, 2019; Accepted: October 09, 2019; Published: October 11, 2019 
Recently, Kim, et al. reported the results of an immunomodulatory toxicity study of AgNPs after oral application at different doses $(60 \mathrm{~nm}$ average size for 28 days). They found that these particles accumulated in rat blood, stomach, lungs, testes, liver, brain, and kidneys without significant genotoxicity [10]. In addition, AgNPs triggered lymphocyte, neutrophil, and eosinophil infiltration into the airways and increased the levels of the interleukin (IL)-13 [11].

\section{Titanium dioxide nanoparticles $\left(\mathrm{TiO}_{2} \mathrm{NPs}\right)$}

Titanium dioxide $\left(\mathrm{TiO}_{2}\right)$ and its derivatives in nanoscale forms are widely used in biomedical and clinical research [12]. These particles have attracted considerable attention for different applications including semiconductors, solar cells, photocatalyst belts, and in consumer products like sunscreen and paints [13-15]. Inhalation, intratracheal instillation, and intranasal application suggest that $\mathrm{TiO}_{2} \mathrm{NPs}_{\text {can }}$ translocate from the nasal cavity into sensory nerves to the nervous system and from the lung into different organs and tissues. $\mathrm{TiO}_{2} \mathrm{NPs}$ alter cell signal transduction pathways, generate reactive oxygen species (ROS), and can be carcinogenic at high doses [16]. Further, inflammation, airway hyperresponsiveness (AHR), IL-18 and IL- $1 \beta$ levels were increased in ovalbumin-sensitized/challenged mice, and these responses were aggravated after $\mathrm{TiO}_{2} \mathrm{NP}$ exposure [17]. The authors also reported that $\mathrm{TiO}_{2} \mathrm{NPs}$ increased the expression of caspase-1 and NLRP3, leading to the production of active caspase-1 in the lung [17]. Additionally, levels of ROS and caspase-1 tended to be augmented in ovalbumin-sensitized/challenged and ovalbuminsensitized/challenged- $\mathrm{TiO}_{2} \mathrm{NP}$-treated mice [17].

\section{Zinc oxide nanoparticles ( $\mathrm{ZnONPs)}$}

Zinc oxide NPs have been widely used in cosmetics, paints, coatings, personal hygiene products, and in biomedicine $[18,19]$. However, exposure to low doses of $\mathrm{ZnONPs}$ induced pulmonary cytotoxicity and inflammation in mice [20].

It has been observed that ZnONPs promoted pulmonary fibrosis, proliferation of airway epithelial cells and goblet cell hyperplasia [21]. IgE levels were up-regulated while IgA levels were down-regulated following ZnONPs exposure [21]. In addition, ZnONPs increased significantly the levels of Th2 cytokines including IL-4, IL-5, and IL-13, and induced significant eosinophilia and neutrophilia in BALF [22]. The specific technique by which $\mathrm{ZnO}$ NPs induced eosinophilic airway inflammation is not completely understood [22].

\section{Copper oxide nanoparticles (CuONPs)}

Copper metal oxide nanoparticles (CuONPs) have been used in different domains including antimicrobial textiles, heat transfer fluid in machines, wood preservation, and catalysts for CO oxidation [23]. In OVA asthma model, CuONPs promoted airway inflammation, cell infiltration into the lung, and mucus secretion [24]. They also increased the phosphorylation of MAPK intracellular signaling pathway components (JNK, Erk, and p38). In addition, these nanoparticles augmented inflammatory cell numbers and increased AHR, ROS, proinflammatory cytokines and IgE levels [24]. This suggests that $\mathrm{CuO}$ NPs can significantly aggravate the evolution of asthma [24].

\section{Silica nanoparticles (SNPs)}

Silica NPs are widely employed in many industrial and scientific area and are considered as a main component of glass and concrete [25]. Occupational exposure to crystalline silica induced airway obstruction, silicosis (a fibrotic lung disease), and lung cancer [26]. Oxidative stress induced by SNPs could injure cells and promote apoptosis [27].
In asthma model, mice exposed to OVA with silica nanoparticles showed a significant increase in AHR and airway inflammation, higher than those treated only with OVA alone [25]. In this study, interferon- $\gamma$, IL-5, IL-1 $\beta$, and IL-13 levels were correlated with the degree of airway inflammation in these animals [25]. Therefore, the airway inflammation was not only induced by the Th2 pathway but also by the Th1 pathway [25].

\section{Graphene oxide nanoparticles (GONPs)}

Graphene oxide NPs (GONPs) are carbonaceous NPs with excellent physicochemical attributes suited to a range of industrial and medical applications. In particular, GONPs might interact with our environment if they make their way into surface water such as lakes or rivers or ground water sources. GONPs have been shown to be strong and sharp enough to penetrate the cell membranes of human lung, skin, and immune cells, leading to several types of tissue damage $[28,29]$.

In a murine model of asthma, GO, used at the sensitization stage, increased airway remodeling and AHR in the form of smooth muscle hypertrophy and goblet cell hyperplasia. However, IL-4, IL-5, and IL-13 levels were decreased in the BALF. Exposure to GO during OVA challenge reduced eosinophil accumulation and augmented macrophage recruitment in the BALF [30]. Moreover, GO treatment elevated the production of the mammalian chitinases CHI3L1 and AMCase in macrophages, whose production is associated with allergic asthma [30]. GO exposure attenuated Th2 responses in OVA-induced asthma and enhanced AHR and remodeling with the induction of mammalian chitinases [30].

\section{Nano-silicon dioxide (nano- $\mathrm{SiO}_{2}$ )}

An essential component of the earth's crust, $\mathrm{SiO}_{2}$ has long been recognized as toxic [31]. However, by virtue of its characteristics such as adjustable pore size and a high specific surface area, $\mathrm{SiO}_{2}$ nanoparticles have recently been employed in biomedicine applications [32]. With the commercial development of nanotechnology, human exposure to nano- $\mathrm{SiO}_{2}$ products has increased. This preliminary research for the first time included the impact of nano- $\mathrm{SiO}_{2}$ on OVAinduced rat asthma. Intratracheal application of nano- $\mathrm{SiO}_{2}$ caused airway remodeling and AHR with or without OVA immunization [33]. Moreover, by increasing nano-SiO, exposure, IL-4 levels in lung homogenate were increased and IFN- $\gamma$ showed a reverse insignificant modulation. Table 1 summarizes the effect of different nanoparticles on airway inflammation.

\section{Carbon nanotubes (CNTs)}

The discovery of carbon nanotubes (CNTs) by Suomi Iijima in 1991 opened up a novel era in materials science. CNTs attracted considerable attention due to their wide potential applications. $\mathrm{CNTs}$ are rolled hexagonal carbon networks capped by half fullerene molecules, less than 100 nanometers in diameter and as thin as 1 or $2 \mathrm{~nm}$ (Figure 1). Recently, they have been used in optics, electronics, and other inventions like medicines and sensor technologies. CNTs form a category of carbon nanomaterials with different structural features to other carbon materials including diamond and graphite [34]. Among several different types of nanomaterials, CNTs are one of the most attractive materials because of their size, strength, and surface chemistry $[35,36]$. In addition, they have a relatively large surface area for chemical modification and can carry a large amount of antigen.

Single-walled CNTs (SWCNTs), double-walled CNTs (DWCNTs), and multi-walled CNTs (MWCNTs) are three common CNT 
Table 1. Nanoparticles and their effects on airway inflammation.

\begin{tabular}{|c|c|c|}
\hline Nanoparticles & Mechanism of action & Effects on airway inflammation \\
\hline Ag & $\begin{array}{l}\text { Evoke neutrophil, lymphocyte and eosinophil infiltration into the airways and elevate the } \\
\text { levels of (Th2) allergic cytokines like IL - } 13 \text {. }\end{array}$ & Stimulate airway inflammation. \\
\hline $\mathrm{TiO}_{2}$ & Increase the expression of IL-1 $\beta$, IL-18, NLRP3 and caspase- 1. & Increase airway inflammation and AHR. \\
\hline ZnO & Enhance the expression of Th2 cytokines and increase IL-5 levels and eosinophil infiltration. & Induce eosinophilic airway inflammation. \\
\hline $\mathrm{CuO}$ & Increase inflammatory cell infiltration into the lung and also pro-inflammatory cytokines. & Induce airway inflammation, AHR, and mucus secretion. \\
\hline $\mathbf{S}$ & $\begin{array}{l}\text { Increase levels of interleukins } \\
\text { IL-5, IL-13, IL-1 } \beta \text {, and interferon- } \gamma \text {. }\end{array}$ & Increase airway inflammation and AHR. \\
\hline GO & Stimulate the production of OVA-specific IgG2a and augment macrophage recruitment. & Augment airway remodeling, AHR, and muscle hypertrophy. \\
\hline $\mathrm{SiO}_{2}$ & Increase the tissue IL- 4 production and Th1/Th2 cytokine imbalance. & Increase airway remodeling and AHR. \\
\hline
\end{tabular}

Various types of carbon nanotubes

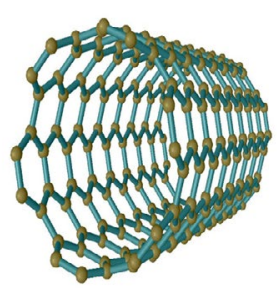

Single walled carbon nanotubes (SWCNTs)
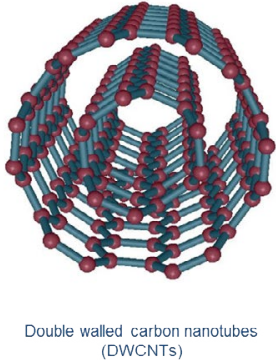

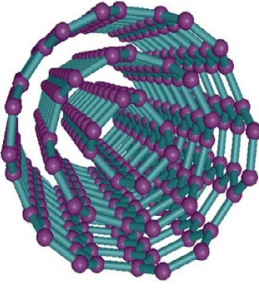

Multi walled carbon nanotubes
(MWCNTs)
Figures 1. Inflammatory cascade and mediators in the pathogenesis of asthmatic inflammation. The inflammation cycle starts from the respiratory epithelium where the antigens, environmental stimuli and nanoparticles can enter and start inducing the inflammatory cells to produce their mediators. These inhaled nanoparticles are able to interact with the immune system and stimulate the production of chemokines and cytokines like interleukin 4 (IL-4), IL-13 and immunoglobulin E (IgE). The released inflammatory mediators cause bronchoconstriction, mucus secretion and lung damage

configurations. The SWCNT structure can be conceptualized by wrapping a one-atom-thick layer of graphite called graphene into a seamless cylinder, while MWCNTs consist of multiple rolled layers (concentric tubes) of graphene. DWCNTs form a special class of nanotubes because their properties and morphology are similar to those of SWNTs but they are more resistant to chemicals.

CNTs can be used as potential carriers for immunization. The conjugation of antigenic epitopes such as viral peptides to CNTs stimulates phagocytosis, rapidly inducing the immune system and improving antibody responses in mice [37].

Recently, it was found that short-term inhalation of the rod-like CNT stimulated a new innate immunity-mediated allergic-like airway inflammation in healthy mice [38]. Investigation of the early stages by transcriptomics showed that exposure to rod-shaped CNTs led to eosinophilia with high expression of Th2-type cytokines, AHR, and mucus hypersecretion [38]. Furthermore, mast cells were used to slightly regulate the airway inflammation caused by rod-like CNTs, and alveolar macrophages were also important participants in the early phases [38].

MWCNTs have many outstanding physical and chemical properties that make them useful in many applications in nanoscience [39]. However, they could trigger allergen-induced inflammation in mouse models, as recognized by the increase in the number of goblet cells in the bronchial epithelium and the infiltration of immune cells (neutrophils, eosinophils, and mononuclear cells) [40].

Another study proposed that airway fibrosis produced by the interaction between inhaled MWCNTs and OVA challenge elevated
IL-5 mRNA levels, which could lead to airway fibrosis [41]. These experiments also showed that MWCNT inhalation needs pre- allergic inflammation to contribute to airway fibrosis, suggesting that persons with pre-existing inflammation might be more susceptible to airway fibrosis from inhaled MWCNTs [41].

Similarly, Li, et al. investigated the effects of SWCNTs in allergic asthma model. Their findings indicated that SWCNTs in rats triggered OVA-induced asthmatic responses [42]. This aggravation was counteracted by the synchronous application of vitamin E [42]. A technique including the downregulation of Th2 reactions, elimination of ROS, and release of allergic asthma symptoms was suggested to explain the antagonistic impact of vitamin $\mathrm{E}$ and provided a preventative therapeutic avenue for asthmatic people exposed to SWCNTs or other nanomaterial-stimulated aggravating activities [42] (Figure 2).

\section{Impact assessment of respiratory exposure to toxic air pollutants}

According to the WHO, air pollution remains a fundamental public and environmental health problem, impacting everyone in developed and developing countries. In spite of improvements in air quality in North America and Western Europe over the last 50 years, mortality and morbidity from air pollution remain high. The WHO estimated that air pollution was accountable for the premature deaths of 3.7 million people under the age of 60 in 2012, and 14\% of deaths were due to chronic obstructive pulmonary disease (COPD) or acute lower respiratory tract infections.

Air pollution is described as contamination by different physical, chemical, or biological agents that modify the natural properties of the atmosphere. Household combustion devices, burning of fossil fuels, motor vehicles, agricultural activities, industrial facilities, and forest fires are common sources of air pollution. Pollutants of major public health concern include particulate matter (PM), carbon monoxide (CO), ozone $\left(\mathrm{O}_{3}\right)$, nitrogen dioxide $\left(\mathrm{NO}_{2}\right)$, and sulfur dioxide $\left(\mathrm{SO}_{2}\right)$. Research into air pollution has recently focused on the new categories of toxicants from micro- and nano-sized particles such as particulate matter (PM) and diesel exhaust (DE) nanoparticles.

\section{Particulate matter (PM)}

PM refers to a mix of fluid droplets and solid particles in the air. Some PM like dirt, dust, bloat, or smoke are large enough to be seen by eye, while others are so small that they can be only visualized by electron microscopy. PMs come in different sizes and shapes and can be released from construction sites, automobile exhaust, fields, industry, smokestacks, and fires.

With urban renovation and evolution, environmental and air pollution have significantly increased and their effect on human 


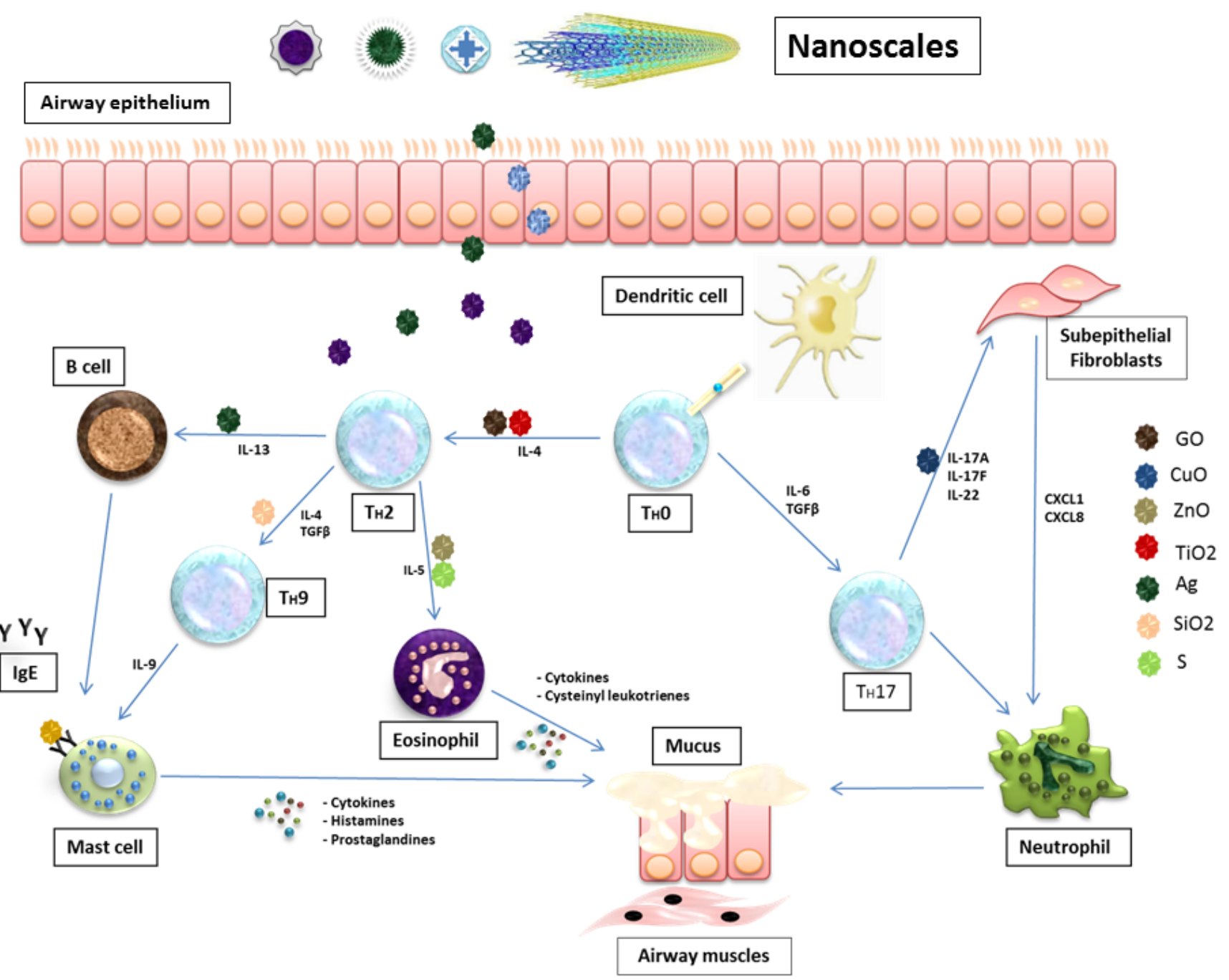

Figures 2. Three common carbon nanotube configurations: single-walled (SWCNTs), double-walled (DWCNTs), and multi-walled (MWCNTs). These incredible structures have an array of unique electronic, magnetic, and mechanical properties

health has attracted renewed research interest. The concept of air pollutants includes PM and gaseous pollutants [43]. Many studies have demonstrated that PM less than $10 \mu \mathrm{m}$ in diameter has a greater effect on human health than larger particles, and an increased PM concentration in the air can accelerate morbidity and mortality at the population level [44]. PM in the atmosphere causes haze, which is not only an annoyance but also dangerously affects human health. PM and its components are closely associated with several cardiovascular and respiratory diseases, including COPD, and asthma [45].

Moreover, exposure to PM during sensitization to allergens has been shown to be sufficient to modify the allergic immune reaction by acting as an adjuvant and facilitating immune memory formation as part of the adaptive immune response [7].

According to American Cancer Society, overall mortality and mortality from cardiopulmonary diseases and lung cancer increased by $4 \%, 6 \%$, and $8 \%$, respectively, for each $10 \mu \mathrm{g} / \mathrm{m}^{3}$ PM2.5 (particles smaller than $2.5 \mu \mathrm{m}$ in diameter) increase. These data were collected from 500,000 adults living in big cities [46]. It has been also reported that PM2.5 can penetrate the lung, irritate the alveolar walls, and decrease lung function [43].

\section{Diesel exhaust (DE) nanoparticles}

DE nanoparticles are defined as a group of PMs derived from diesel fossil fuels and combustion engines. Small particles released from diesel exhaust (DEP) are associated with different pulmonary and cardiovascular pathologies like asthma and pulmonary fibrosis [4750]. In the 1990s, several epidemiological studies reported an increase in asthma incidence in urban areas. Moreover, respiratory toxicity characterized by airway inflammation, lung fibrosis, AHR, and goblet cell hyperplasia was observed in a mouse model after long-term DEP exposure [51].

Recently, an ovalbumin (OVA) mouse model of asthma was used to investigate the effects of intranasal administration of diesel exhaust particles. Mice exposed to DEPs were more hypersensitive to ovalbumin than controls as demonstrated by AHR, airway inflammation, and ovalbumin-specific IgE levels [52]. In another study, the impact of DEP treatment on asthma severity was studied in a house dust mite (HDM) mouse model. DEP exposure alone did not stimulate an asthmatic reaction, while co-exposure (DEP-HDM) increased airway hyperresponsiveness compared with HDM exposure alone [53]. 
The authors of the previous studies concluded that smog including PMs increased the likelihood of suffering from asthma, respiratory tract infections, and influenza. Therefore, reducing the impact of air pollution and its PMs on respiratory health is highly topical issue and a challenging task.

\section{Conclusion}

Asthma is a chronic obstructive disease associated with chronic airway inflammation that results in symptoms like a temporary airway narrowing with shortness of breath, wheezing, cough, and chest tightness. Nanoparticles are a promising instrument for future therapeutic strategies due to their unique properties. However, they can also lead to harmful diseases and trigger asthmatic inflammation. Toxicity and safety studies like neurotoxicity, carcinogenicity, and immunotoxicity must be performed on each individual NP to investigate harmful and/or undesirable outcomes. The physiochemical characterization of NPs including their administration, distribution, metabolism, and excretion should be also analyzed to improve safety and efficacy. When such analyses have been examined and validated, the product can be transmitted from the preclinical to clinical phase and applied to humans.

The impact of air pollution and its components like PM have attracted worldwide attention in attempts to limit our body's exposure to harmful substances. This review focused on the ongoing studies of different types of nanoparticles given their leading role in airway inflammation and summarized some in vivo experiments as an initial step towards clinical translation.

\section{Authors' contributions}

SAH performed the literature search and wrote the manuscript. JN, $\mathrm{AM}$ and $\mathrm{SH}$ assisted in writing the final version of the manuscript. $\mathrm{RB}$ read and approved the final manuscript. QTD conceived and conceptualized the manuscript. All authors read and approved the manuscript.

\section{Consent for publication}

Not applicable.

\section{Competing interests}

The authors declare that they have no competing interests.

\section{References}

1. Omlor AJ, Le DD, Schlicker J, Hannig M, Ewen R, et al. (2017) Local Effects on Airway Inflammation and Systemic Uptake of $5 \mathrm{~nm}$ PEGylated and Citrated Gold Nanoparticles in Asthmatic Mice. Small p. 13.

2. Gatti AM, Kirkpatrick J, Gambarelli A, Capitani F, Hansen T, et al. (2008) ESEM evaluations of muscle/nanoparticles interface in a rat model. J Mater Sci Mater Med 19: $1515-1522$.

3. Cho EC, Zhang Q, Xia Y (2011) The effect of sedimentation and diffusion on cellular uptake of gold nanoparticles. Nat Nanotechnol 6: 385-391.

4. Podila R, Brown JM (2013) Toxicity of engineered nanomaterials: a physicochemical perspective. J Biochem Mol Toxicol 27: 50-55.

5. da Silva AL, Cruz FF, Rocco PRM, Morales MM (2017) New perspectives in nanotherapeutics for chronic respiratory diseases. Biophys Rev 9: 793-803.

6. Heck S, Nguyen J, Le DD, Bals R, Dinh QT (2015) Pharmacological Therapy of Bronchial Asthma: The Role of Biologicals. Int Arch Allergy Immunol 168: 241-252.

7. Castaneda AR, Pinkerton KE (2016) Investigating the Effects of Particulate Matter on House Dust Mite and Ovalbumin Allergic Airway Inflammation in Mice. Curr Protoc Toxicol 68: 18 .

8. Mittal AK, Chisti Y, Banerjee UC (2013) Synthesis of metallic nanoparticles using plant extracts. Biotechnol Adv 31: 346-356.
9. Pugazhendhi S, Sathya P, Palanisamy PK, Gopalakrishnan R (2016) Synthesis of silver nanoparticles through green approach using Dioscorea alata and their characterization on antibacterial activities and optical limiting behavior. J Photochem Photobiol B 159: 155-160.

10. Kim YS, Kim JS, Cho HS, Rha DS, Kim JM, et al. (2008) Twenty-eight-day oral toxicity, genotoxicity, and gender-related tissue distribution of silver nanoparticles in Sprague-Dawley rats. Inhal Toxicol 20: 575-583.

11. Chuang HC, Hsiao TC, Wu CK, Chang HH, Lee CH, et al. (2013) Allergenicity and toxicology of inhaled silver nanoparticles in allergen-provocation mice models. Int $J$ Nanomedicine 8: 4495-4506.

12. Oliveira VR, Uriarte JJ, Falcones B, Jorba I, Zin WA, et al. (2019) Biomechanical Response of Lung Epithelial Cells to Iron Oxide and Titanium Dioxide Nanoparticles. Front Physiol 10: 1047.

13. Lomer MC, Thompson RP, Powell JJ (2002) Fine and ultrafine particles of the diet influence on the mucosal immune response and association with Crohn's disease. Proc Nutr Soc 61: 123-130.

14. Gelis C, Girard S, Mavon A, Delverdier M, Paillous N, et al. (2003) Assessment of the skin photoprotective capacities of an organo-mineral broad-spectrum sunblock on two ex vivo skin models. Photodermatol Photoimmunol Photomed 19: 242-253.

15. Sahu KK, Alex TC, Mishra D, Agrawal A (2006) An overview on the production of pigment grade titania from titania-rich slag. Waste Manag Res 24: 74-79.

16. Shi H, Magaye R, Castranova V, Zhao J (2013) Titanium dioxide nanoparticles: a review of current toxicological data. Part Fibre Toxicol 10: 15

17. Kim BG, Lee PH, Lee SH, Park MK, Jang AS (2017) Effect of $\mathrm{TiO}_{2}$ Nanoparticles on Inflammasome-Mediated Airway Inflammation and Responsiveness. Allergy Asthma Immunol Res 9: 257-264.

18. Fine JM, Gordon T, Chen LC, Kinney P, Falcone G, et al. (1997) Metal fume fever: characterization of clinical and plasma IL-6 responses in controlled human exposures to zinc oxide fume at and below the threshold limit value. J Occup Environ Med 39: $722-726$.

19. Rasmussen JW, Martinez E, Louka P, Wingett DG (2010) Zinc oxide nanoparticles for selective destruction of tumor cells and potential for drug delivery applications. Expert Opin Drug Deliv 7: 1063-1077.

20. Hadrup N, Rahmani F, Jacobsen NR, Saber AT, Jackson P, et al. (2019) Acute phase response and inflammation following pulmonary exposure to low doses of zinc oxide nanoparticles in mice. Nanotoxicology 1-18.

21. Cho WS, Duffin R, Howie SE, Scotton CJ, Wallace WA, et al. (2011) Progressive severe lung injury by zinc oxide nanoparticles; the role of $\mathrm{Zn}^{2+}$ dissolution inside lysosomes. Part Fibre Toxicol 8: 27.

22. Huang KL, Lee YH, Chen HI, Liao HS, Chiang BL, et al. (2015) Zinc oxide nanoparticles induce eosinophilic airway inflammation in mice. J Hazard Mater 297: 304-312.

23. Lai X, Zhao H, Zhang Y, Guo K, Xu Y, et al. (2018) Intranasal Delivery of Copper Oxide Nanoparticles Induces Pulmonary Toxicity and Fibrosis in C57BL/6 mice. Sci Rep 8: 4499.

24. Park JW, Lee IC, Shin NR, Jeon CM, Kwon OK, et al. (2016) Copper oxide nanoparticles aggravate airway inflammation and mucus production in asthmatic mice via MAPK signaling. Nanotoxicology 10: 445-452.

25. Park HJ, Sohn JH, Kim YJ, Park YH, Han H, et al. (2015) Acute exposure to silica nanoparticles aggravate airway inflammation: different effects according to surface characteristics. Exp Mol Med 47: e173.

26. Leung CC, Yu IT, Chen W (2012) Silicosis. Lancet 379: 2008-2018.

27. Fu PP, Xia Q, Hwang HM, Ray PC, Yu H (2014) Mechanisms of nanotoxicity: generation of reactive oxygen species. J Food Drug Anal 22: 64-75.

28. Ahmed F, Rodrigues DF (2013) Investigation of acute effects of graphene oxide on wastewater microbial community: a case study. J Hazard Mater 256-257: 33-39.

29. Duch MC, Budinger GR, Liang YT, Soberanes S, Urich D, et al. (2011) Minimizing oxidation and stable nanoscale dispersion improves the biocompatibility of graphene in the lung. Nano Lett 11: 5201-5207.

30. Shurin MR, Yanamala N, Kisin ER, Tkach AV, Shurin GV, et al. (2014) Graphene oxide attenuates Th2-type immune responses, but augments airway remodeling and hyperresponsiveness in a murine model of asthma. ACS Nano 8: 5585-5599. 
31. Ding M, Chen F, Shi X, Yucesoy B, Mossman B, et al. (2002) Diseases caused by silica: mechanisms of injury and disease development. Int Immunopharmacol 2: 173182.

32. Suh WH, Suslick KS, Stucky GD, Suh YH (2009) Nanotechnology, nanotoxicology, and neuroscience. Prog Neurobiol 87: 133-170.

33. Han B, Guo J, Abrahaley T, Qin L, Wang L, et al. (2011) Adverse effect of nano-silicon dioxide on lung function of rats with or without ovalbumin immunization. PLoS One 6: e17236.

34. De Volder MF, Tawfick SH, Baughman RH, Hart AJ (2013) Carbon nanotubes: present and future commercial applications. Science 339: 535-539.

35. Sun YP, Fu K, Lin Y, Huang W (2002) Functionalized carbon nanotubes: properties and applications. Acc Chem Res 35: 1096-1104.

36. Mitchell LA, Gao J, Wal RV, Gigliotti A, Burchiel SW, et al. (2007) Pulmonary and systemic immune response to inhaled multiwalled carbon nanotubes. Toxicol Sci 100: 203-214.

37. Pantarotto D, Partidos CD, Hoebeke J, Brown F, Kramer E, et al. (2003) Immunization with peptide-functionalized carbon nanotubes enhances virus-specific neutralizing antibody responses. Chem Biol 10: 961-966.

38. Rydman EM, Ilves M, Koivisto AJ, Kinaret PA, Fortino V, et al. (2014) Inhalation of rod-like carbon nanotubes causes unconventional allergic airway inflammation. Part Fibre Toxicol 11: 48

39. Di CL, Bianchi MG, Chiu M, Taurino G, Donato F, et al. (2009) Comparative in Vitro Cytotoxicity of Realistic Doses of Benchmark Multi-Walled Carbon Nanotubes towards Macrophages and Airway Epithelial Cells. Nanomaterials p. 9.

40. Inoue K, Koike E, Yanagisawa R, Hirano S, Nishikawa M, et al. (2009) Effects of multi-walled carbon nanotubes on a murine allergic airway inflammation model. Toxicol Appl Pharmacol 237: 306-316.

41. Ryman-Rasmussen JP, Tewksbury EW, Moss OR, Cesta MF, Wong BA, et al. (2009) Inhaled multiwalled carbon nanotubes potentiate airway fibrosis in murine allergic asthma. Am J Respir Cell Mol Biol 40: 349-358.
42. Li J, Li L, Chen H, Chang Q, Liu X, et al. (2014) Application of vitamin E to antagonize SWCNTs-induced exacerbation of allergic asthma. Sci Rep 4: 4275.

43. Xing YF, Xu YH, Shi MH, Lian YX (2016) The impact of PM2.5 on the human respiratory system. J Thorac Dis 8: E69-E74.

44. Nemery B, Hoet PH, Nemmar A (2001) The Meuse Valley fog of 1930: an air pollution disaster. Lancet 357: 704-708

45. Cao LM, Zhou Y, Zhang Z, Sun WW, Mu G, et al. (2016) Impacts of airborne particulate matter and its components on respiratory system health. 50: 1114-1118.

46. Pope CA III, Burnett RT, Thun MJ, Calle EE, Krewski D, et al. (2002) Lung cancer, cardiopulmonary mortality, and long-term exposure to fine particulate air pollution. JAMA 287: 1132-1141.

47. Donaldson K, Tran L, Jimenez LA, Duffin R, Newby DE, et al. (2005) Combustionderived nanoparticles: a review of their toxicology following inhalation exposure. Part Fibre Toxicol 2: 10 .

48. Nel A (2005) Atmosphere. Air pollution-related illness: effects of particles. Science 308: 804-806.

49. Nemmar A, Al-Maskari S, Ali BH, Al-Amri IS (2007) Cardiovascular and lung inflammatory effects induced by systemically administered diesel exhaust particles in rats. Am J Physiol Lung Cell Mol Physiol 292: L664-L670.

50. Beelen R, Hoek G, van den Brandt PA, Goldbohm RA, Fischer P, et al. (2008) Longterm exposure to traffic-related air pollution and lung cancer risk. Epidemiology 19: $702-710$

51. Kim BG, Lee PH, Lee SH, Kim YE, Shin MY, et al. (2016) Long-Term Effects of Diesel Exhaust Particles on Airway Inflammation and Remodeling in a Mouse Model Allergy Asthma Immunol Res 8: 246-256.

52. Manners S, Alam R, Schwartz DA, Gorska MM (2014) A mouse model links asthma susceptibility to prenatal exposure to diesel exhaust. J Allergy Clin Immunol 134: 63-72.

53. Brandt EB, Kovacic MB, Lee GB, Gibson AM, Acciani TH, et al. (2013) Diesel exhaust particle induction of IL-17A contributes to severe asthma. $J$ Allergy Clin Immunol 132: 1194-1204.

Copyright: (C2019 Harfoush SA. This is an open-access article distributed under the terms of the Creative Commons Attribution License, which permits unrestricted use, distribution, and reproduction in any medium, provided the original author and source are credited. 